\title{
Controlled Positioning of Nanoparticles on Graphene by Noninvasive AFM Lithography
}

\author{
Elena Bellido, ${ }^{\dagger}$ Isaac Ojea-Jiménez, ${ }^{\dagger}$ Alberto Ghirri, ${ }^{\S}$ Christian Alvino, ${ }^{\S}, \|$ Andrea Candini, ${ }^{\S}$ \\ Victor Puntes, ${ }^{\dagger, \ddagger}$ Marco Affronte, ${ }^{\S, \|}$ Neus Domingo, ${ }^{*}, \dagger$ and Daniel Ruiz-Molina ${ }^{*}, \dagger$ \\ †Centro de Investigación en Nanociencia y Nanotecnología (CIN2, ICN-CSIC) Esfera UAB, Campus UAB, Cerdanyola del Vallès, \\ 08193 Spain \\ ${ }^{\ddagger}$ Institut Català de Recerca i Estudis Avançats (ICREA) 08093, Barcelona Spain \\ ${ }^{\S}$ S3 Centre, Istituto Nanoscienze-CNR, via Campi 213/a, 41100 Modena, Italy \\ "Dipartimento di Fisica, Università di Modena e Reggio Emilia, via Campi 213/a, 41100 Modena, Italy
}

ABSTRACT: Atomic force microscopy is shown to be an excellent lithographic technique to directly deposit nanoparticles on graphene by capillary transport without any previous functionalization of neither the nanoparticles nor the graphene surface while preserving its integrity and conductivity properties. Moreover this technique allows for (sub)micrometric control on the positioning thanks to a new three-step protocol that has been designed with this aim. With this methodology the exact target coordinates are registered by scanning the tip over the predetermined area previous to its coating with the ink and deposition. As a proof-of-concept, this strategy has successfully allowed the controlled deposition of few nanoparticles on $1 \mu \mathrm{m}^{2}$ preselected sites of a graphene surface with high accuracy.

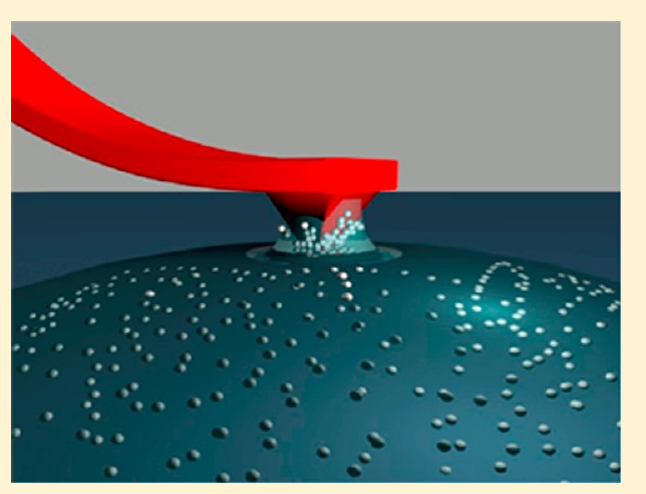

\section{INTRODUCTION}

Since its discovery in 2004, ${ }^{1}$ graphene has raised much attention due to its interesting electrical, optical, thermal, and

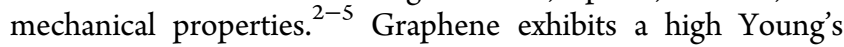
modulus and fracture strength with potential applications in reinforced composites. ${ }^{6}$ Its high thermal/chemical stability and large specific surface area are also of interest in catalysis and sensors, ${ }^{7}$ whereas the interesting transport phenomena and high optical transmittance are of great relevance in photonics and optoelectronics, ${ }^{8}$ as well as in electronics and spintronics. $^{9-12}$ Most of these applications require the development of graphene-based hybrid systems in order to enable versatile and tailor-made properties with performances far beyond those of the individual materials. ${ }^{13}$ Although the accurate incorporation of different families of organic and/or inorganic materials without modifying the graphene intrinsic structure and properties is a delicate step, to date, this challenge has been addressed by means of either in situ syntheses ${ }^{14-16}$ or selfassembly processes, ${ }^{17,18}$ which permitted the functionalization of graphene with a wide variety of materials, such as organic compounds, polymers, inorganic nanoparticles (NPs), and other molecular-based materials. ${ }^{7}$ For instance, Bendiab et al. grafted by drop casting deposition a rare-earth-based bis(phthalocyaninato) complex tailored with pyrenyl groups that favor the $\pi-\pi$ stacking with the graphene surface. ${ }^{11} \mathrm{Au}$ nanostructures deposited on graphene by thermal evaporation or by a combination of electron beam, metallic film deposition, and lift-off processes have also been shown to enhance surface enhanced Raman spectroscopy (SERS) ${ }^{19}$ and Raman responses, ${ }^{20}$ respectively. In addition, the optical and electrical properties of graphene can also be tailored by the site-specific patterning of $\mathrm{Au}$ NPs decorated with cinnamate moieties covalently cross-linked by UV irradiation. ${ }^{21}$ Although these studies were successful on structuring materials on graphene with control on feature thickness and size, the ability to accurately control the positioning of these structures on preselected sites of a graphene surface by a mild method still remains a challenge. If successful, this will open new perspectives mainly for device and sensing applications, where objects need to be placed with high precision onto well-defined sensing regions of the device. ${ }^{22}$

In this article, we demonstrate that direct-write atomic force microscopy (AFM) lithography represents an excellent approach to this aim. In a typical experiment a probe tip is coated with a solution containing the NPs (commonly referred to as ink solution) and used as a carrier system to deposit it on a graphene surface, as done in the so-called dip-pen nanolithography (DPN) technique. ${ }^{23-25}$ The ink is delivered in direct contact with the unmodified graphene surface, while ensuring that the integrity of graphene is preserved. To demonstrate the viability of this approach we have selected cobalt nanoparticles (Co-NPs) uniform in size and morphol-

Received: January 23, 2012

Revised: July 10, 2012

Published: July 25, 2012 
ogy, ${ }^{26}$ whose deposition has become a challenge since most methodologies so far used with this aim need high temperature treatments. ${ }^{27}$ The mild processing conditions (lack of electric irradiation), ease of use, and accessibility are also strong potential advantages of this AFM-based approach. Even the positioning capabilities of this technique have previously been exploited to fabricate electrical contacts on single graphene flakes, ${ }^{28}$ it has never been used for the direct deposition of NPs on graphene.

\section{EXPERIMENTAL SECTION}

2.1. Materials. Cobalt carbonyl $\left[\mathrm{Co}_{2}(\mathrm{CO})_{8}\right.$, moistened with 5$10 \%$ hexane], trioctylphosphine oxide (TOPO, 99\%), oleic acid (99\%), hexane anhydrous (95\%), toluene anhydrous (99.8\%), and 1,2dichlorobenzene anhydrous (o-DCB, 99\%) were purchased from Sigma-Aldrich and used as received without further purification. Synthesis of NPs was carried out under Ar-atmosphere conditions using the standard Schlenk line setup. Single pen (SP) tips are commercial silicon nitride Type A single pen (NanoInk, Inc., Chicago, IL) with a spring constant of $\sim 0.1 \mathrm{~N} \mathrm{~m}^{-1}$. Noncontact high-resonance frequency $(\mathrm{NCH})$ tips are PPP-NCH silicon point probes with a spring constant of $\sim 42 \mathrm{~N} \mathrm{~m}^{-1}$ and resonant frequency of $\sim 330 \mathrm{kHz}$, purchased from Nanosensors.

2.2. Preparation of the Ink Solution. The ink solution used for these experiments consisted in a colloidal solution of monodispersed oleic acid-stabilized Co-NPs $\left(2 \times 10^{16} \mathrm{NPs} \mathrm{mL}, \sim 40 \mathrm{mg} \mathrm{mL}^{-1}\right)$ of a mean size of $7.6 \pm 1.0 \mathrm{~nm}$ diameter prepared following a previous procedure. $^{26}$ Briefly, TOPO $(0.1 \mathrm{~g})$ and oleic acid $(0.2 \mathrm{~mL})$ were degassed in argon in a flask for $20 \mathrm{~min}$. Then, $15 \mathrm{~mL}$ of $o$-DCB was introduced into the flask under an argon atmosphere. The solution was heated to the reflux temperature of $o$-DCB $\left(182{ }^{\circ} \mathrm{C}\right)$ and $0.54 \mathrm{~g}$ of $\mathrm{Co}_{2}(\mathrm{CO})_{8}$ diluted in $3 \mathrm{~mL}$ of $o$-DCB was quickly injected into the mixture. The reaction continued for another $10 \mathrm{~min}$, and then the black colloidal solution was extracted using an airtight syringe and stored in a glass vial under argon. NP morphology and size distribution was determined by transmission electron microscopy (TEM) analysis on samples prepared by dropping the NP solution onto a carboncoated TEM grid. The solvent was allowed to evaporate under air and the mean size was verified by TEM (Figure 1). The typical epsilon (cubic) crystal anisotropy ( $\varepsilon$-Co-NPs) together with absence of

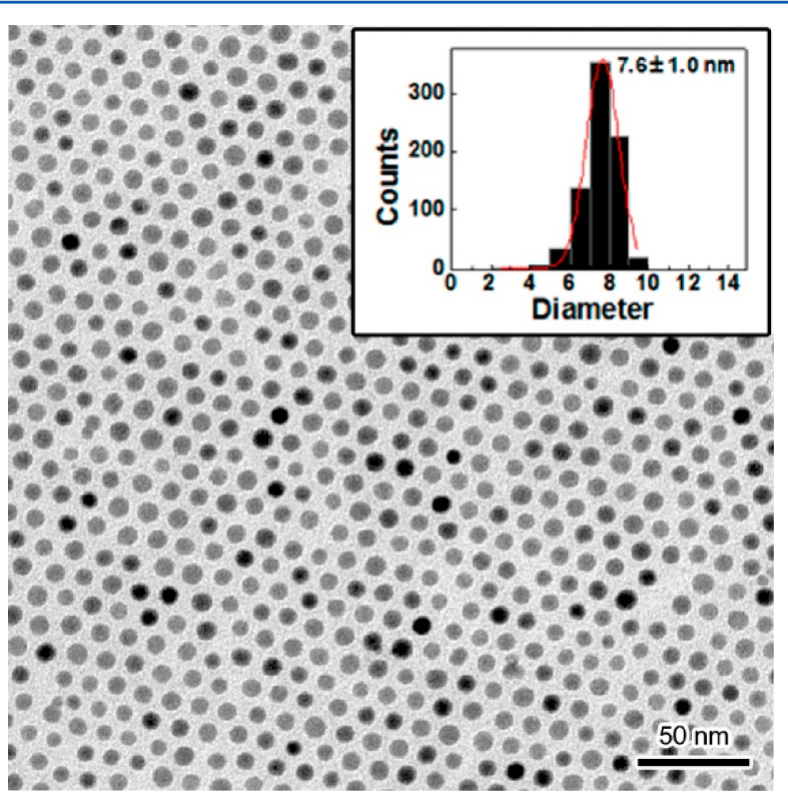

Figure 1. Morphological analysis (TEM) of a monolayer of oleic acidcoated Co-NPs with a hexagonal packing. The inset shows the mean size distribution of Co-NP measured from TEM images. oxidation was confirmed by X-ray diffraction spectroscopy (XRD). For further deposition experiments, the as-synthesized Co-NPs $(2 \mathrm{~mL})$ were washed by precipitation with anhydrous methanol $(2 \mathrm{~mL})$ followed by redispersion into a solution of either hexane, toluene, or $o$ DCB $(20 \mathrm{~mL})$ containing $0.08 \%(\mathrm{w} / \mathrm{w})$ oleic acid. The solutions were stored under inert conditions and were stable before the deposition experiments.

2.3. Preparation of the Target Substrates. Silicon bearing native oxide layer $\left(\mathrm{Si} / \mathrm{SiO}_{2}\right)$ substrates were prepared by initially cutting silicon wafers into $0.5 \times 0.5 \mathrm{~cm}^{2}$ pieces. Then, each $\mathrm{Si} / \mathrm{SiO}_{2}$ substrate was washed in an ultrasonic bath for $10 \mathrm{~min}$ in acetonitrile, ethanol, and Milli-Q water and dried by blowing nitrogen gas. Highly ordered pyrolytic graphite (HOPG) surfaces were purchased from SPI suppliers. HOPG was cleaved with adhesive tape to obtain fresh thin film samples. Carbon-coated TEM grids, carbon type-B, 200 mesh copper grids were purchased from Ted Pella, Inc. Graphene flakes were obtained by the mechanical exfoliation method from natural graphite (NGS GmbH, Germany). Thin flakes were optically located with respect to prepatterned alignment markers on top of p-doped Silicon wafer coated with $300 \mathrm{~nm}$ of oxide $\left(\mathrm{SiO}_{2}\right)$. The effective number of layers was checked by micro-Raman spectroscopy. Raman measurements were performed using a Jobin-Yvon LabRam spectrometer with a $\mathrm{He}-\mathrm{Ne}$ laser at $632.8 \mathrm{~nm}$ wavelength. The incident power was $\sim 20 \mathrm{~mW}$. Metal contacts $(\mathrm{Ti} / \mathrm{Pt} 10 / 100 \mathrm{~nm})$ on the graphene sheets have been obtained by electron beam lithography (EBL), electron-beam evaporation, and lift-off. The underlying doped wafer was contacted from the bottom and used as the backgate. The patterning of the flakes in cross-like geometry was performed by means of EBL. The uncovered parts of the sample were removed by a $50 \mathrm{~W}$ oxygen plasma. Electrical measurements were performed by using the lock-in technique with an applied ac current $(51 \mathrm{nA}$ at $33.77 \mathrm{~Hz})$ in a liquid-He cryostat with a base temperature $2 \mathrm{~K}$ and equipped with a superconducting magnet with a field as high as $7 \mathrm{~T}$.

2.4. AFM Lithography Experiments: Tip Functionalization and Deposition Procedure. Tips were coated with the ink solution containing the NPs via the scanning coating method as follows. ${ }^{29,30}$ The process is schematically depicted in Figure 2a. First, a drop of the Co-NP-based ink solution was deposited by drop casting onto an auxiliary $\mathrm{Si} / \mathrm{SiO}_{2}$ substrate and the solution was left to partially evaporate for a given time until it remained a very thin liquid film at the edge of the droplet. Then, the tip was approached to the surface until it touched the film. Once in contact, it was scanned over a $5 \times 5$ $\mu \mathrm{m}^{2}$ area at $1 \mathrm{~Hz}$, while keeping the tip dipped into the ink film. In the case of the SP tips, it was necessary to reduce the excess of ink (Co-

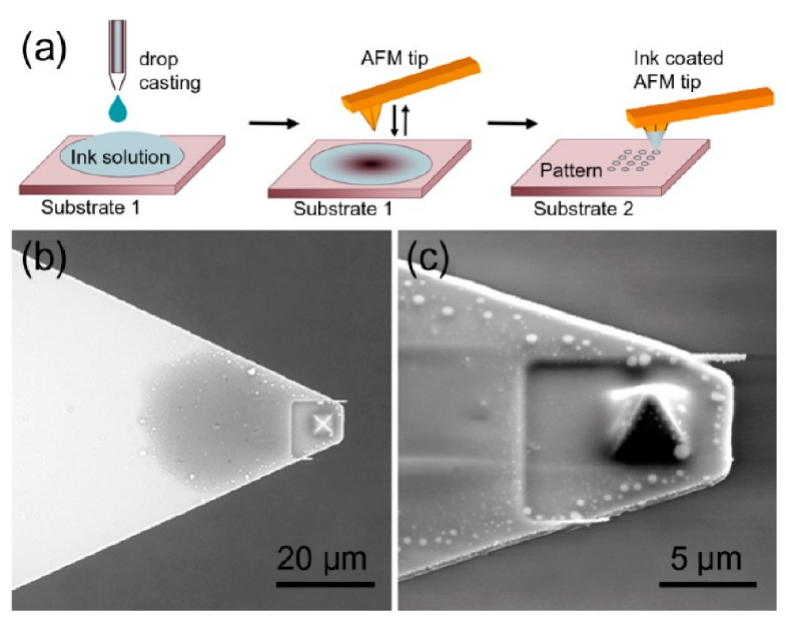

Figure 2. (a) Schematic illustration of the scanning coating method for the functionalization of the AFM tip with the ink solution and subsequent deposition of the solution onto a surface in the form of the desired pattern. FE-SEM image of a SP tip loaded with the ink solution [Co-NPs $\left(2 \times 10^{15} \mathrm{NPs} \mathrm{mL}^{-1}\right)$ in $o$-DCB with an excess of oleic acid $(0.08 \% \mathrm{w} / \mathrm{w})]$ viewed $(\mathrm{b})$ from above and $(\mathrm{c})$ at $45^{\circ}$ tilt angle. 
NP-based solution) on the tip. This was achieved by bringing the freshly coated SP-tip in contact with a $\mathrm{Si} / \mathrm{SiO}_{2}$ substrate and creating spots with an average diameter of several micrometers, which quickly decreased in size. After having deposited a few micrometer-sized spots, it is possible to start writing uniform dots in a very controllable manner. Conversely, this step was not necessary for $\mathrm{NCH}$ tips due to their initial low ink loading.

All of the experiments using an SP tip were performed with an Nscriptor DPN System (NanoInk, Inc., Chicago, IL). Environmental conditions of $\sim 50 \%$ relative humidity and room temperature were kept constant during all DPN patterning processes with an integrated environmental chamber as part of the Nscriptor DPN System. All of the experiments involving $\mathrm{NCH}$ tips were performed with an Agilent 5500 AFM/SPM microscope (formerly Molecular Imaging PicoPlus $\mathrm{AFM}$ ). A multipurpose closed loop $\mathrm{X}-\mathrm{Y}$ scanner with an $\mathrm{X}-\mathrm{Y}$ nanopositioning stage (NPXY100E from nPoint) and a scan range up to $100 \times 100 \mu \mathrm{m}^{2}$ was used for the deposition of Co-NPs under controlled environmental conditions $(\sim 50 \%$ relative humidity and room temperature).

2.5. Characterization Methods. TEM analysis of NPs size distribution was performed on a JEOL1010 transmission electron microscope at an accelerating voltage of $80 \mathrm{kV}$. Digital images were analyzed with the ImageJ software and a custom macro performing smoothing $(3 \times 3$ or $5 \times 5$ median filter $)$, manual global threshold, and automatic particle analysis provided by the ImageJ. The macro can be downloaded from http://code.google.com/p/psa-macro. TEM images of NPs deposited with a tip were taken with a JEOL JEM2100 microscope (JEOL LTD, Tokio, Japan). XRD data were collected on a PANalytical X́Pert diffractometer using a Co $\mathrm{K} \alpha$ radiation source $(\lambda=1.789 \AA)$. In a typical experiment, the $2 \theta$ diffraction (Bragg) angles were measured by scanning the goniometer from $25^{\circ}$ to $60^{\circ}$ at a speed of $0.021^{\circ} \mathrm{s}^{-1}$. The samples were prepared by precipitating as-synthesized Co-NPs $(2 \mathrm{~mL})$ in the presence of methanol and dried under argon before smearing onto (510) silicon wafers $\left(3^{\circ}\right.$ off axis) for XRD analysis. Dynamic noncontact mode AFM images of NP depositions were recorded on an Agilent 5500 AFM/ SPM microscope using $\mathrm{NCH}$ tips. AFM image processing and rendering was done with Gwyddion data analysis software.

\section{RESULTS AND DISCUSSION}

3.1. Nanoparticle Structuration on Bare $\mathrm{Si} / \mathrm{SiO}_{2}$. CoNPs of $7.6 \pm 1.0 \mathrm{~nm}$ in diameter stabilized with an oleic acid capping layer were selected as model nanometric system for their deposition. ${ }^{26}$ A morphological TEM image of a hexagonal packed monolayer of such oleic acid-coated Co-NPs together with their corresponding mean size distribution is shown in Figure 1.

In a typical experiment, the Co-NPs were dispersed in organic media at $2 \times 10^{15} \mathrm{NPs} \mathrm{mL}^{-1}$ and the solution used as base ink for the deposition experiments on bare $\mathrm{Si} / \mathrm{SiO}_{2}$ substrates by using single pen (SP) tips. These are AFM tips with a symmetric pyramidal shape and low aspect ratio specially designed with a recessed area surrounding the tip base that provides a higher ink material loading, and therefore, a long stable writing performance. Initially, SP tips were coated with the ink solution via the scanning coating method schematically depicted in Figure 2a (see the Experimental Section for details), which ensured an efficient loading of the ink on the tip as verified using field-emission scanning electron microscopy (FESEM; Figure 2b,c).

Once the tips were coated, the transference of the material in the form of dot patterns occurred through the delivery of less than femtoliter droplets of the ink while controlling the tipsubstrate contact time. In this methodology, the solvent plays an important role and should fulfill specific requirements to ensure that the ink is appropriate for the deposition process.
Specifically, the ink should exhibit (I) a proper viscosity to ensure not only an homogeneous coating on the tip but also a controlled transference of the material, (II) a high boiling point to maintain the liquid state over the whole writing process, and (III) a proper solvent-surface wettability balance. While a very polar solvent will spread over the polar $\mathrm{Si} / \mathrm{SiO}_{2}$ surface making any structuration difficult, the use of nonpolar solvents will induce the formation of droplets with very high contact angles thus hampering the transfer of the material. In this work, we have faced this challenge by the examination of three different organic solvents (hexane, toluene, and o-dichlorobenzene) of varying boiling points and low polarities that ensure a good contact angle with the $\mathrm{Si} / \mathrm{SiO}_{2}$ surface (a constant relative humidity and temperature of $50 \%$ and $23{ }^{\circ} \mathrm{C}$, respectively, was used in all the cases to allow for a proper comparison). Initially, the use of hexane as solvent significantly hampered the deposition process due to its highly volatile character (bp 69 ${ }^{\circ} \mathrm{C}$ ), since it rapidly evaporated during the tip coating step. By contrast, the use of toluene (bp $110^{\circ} \mathrm{C}$ ) partially slowed down the drying rate of the ink solution, allowing an appropriate coating of the tip and the subsequent deposition of NPs in the form of arrays (Figure 3a). However, after fabrication of few arrays, the ink solution unavoidably dried on the tip surface leading to the deposition of broken solid pieces on the surface during the writing process. Finally, the use of $o$-dichlorobenzene $(o-\mathrm{DCB})$ in the ink solution was shown to be the most convenient solvent, allowing the obtaining of well-defined arrays made of repeated structures with control on their morphology and size down to $500 \mathrm{~nm}$. The topographic profile of a representative array, shown in Figure $3 b$, evidenced the high uniformity reached over an entire array made of 100 repeated dots. The height of the structures was $12 \pm 1 \mathrm{~nm}$, which is in good agreement with that expected for a monolayer of Co-NPs.

Part of the success with $o$-DCB comes from its high boiling point (bp $180{ }^{\circ} \mathrm{C}$ ) that increases the total drying time of the ink, favoring a stable transference of the NPs to the substrate over an extended period of time. Additionally, oleic acid molecules, either coating the NP surface and freely dispersed in the solution $(0.08 \% \mathrm{w} / \mathrm{w})$, also contributed to maintain the ink in a liquid state for extended times and to increase the viscosity of the ink solution. ${ }^{31,32}$ The effective transference of NPs under these experimental conditions was confirmed with a control experiment where two different inks were used to make arrays: one consisting of an $o$-DCB solution free of NPs but with a $0.08 \% \mathrm{w} / \mathrm{w}$ oleic acid that emulates the concentration present in the original ink and a second one with the same conditions but containing NPs. Both inks were used under exactly the same experimental conditions to generate similar arrays on $\mathrm{Si}$ / $\mathrm{SiO}_{2}$, and the obtained structures were compared by AFM analysis. Whereas the average height of the arrays made with the NP-based ink was $10 \pm 1 \mathrm{~nm}$, the array fabricated with the NP-free ink solution was $3 \pm 1 \mathrm{~nm}$ in height for dots of similar diameter ( $559 \pm 29 \mathrm{~nm}$ and $473 \pm 51 \mathrm{~nm}$, respectively, Figure $3 \mathrm{c}, \mathrm{d})$. The decrease in height of the pattern was clearly related to the absence of NPs in the last case, while the background height was attributed to the presence of remaining oleic acid molecules from the ink solution.

3.2. Nanoparticle Deposition on Single-Layer Graphene. The optimized experimental conditions described above were used first for the deposition of a NPs-free ink on single-layer graphene as well as on the surrounding exposed areas of the supporting $\mathrm{Si} / \mathrm{SiO}_{2}$ substrate to check the 

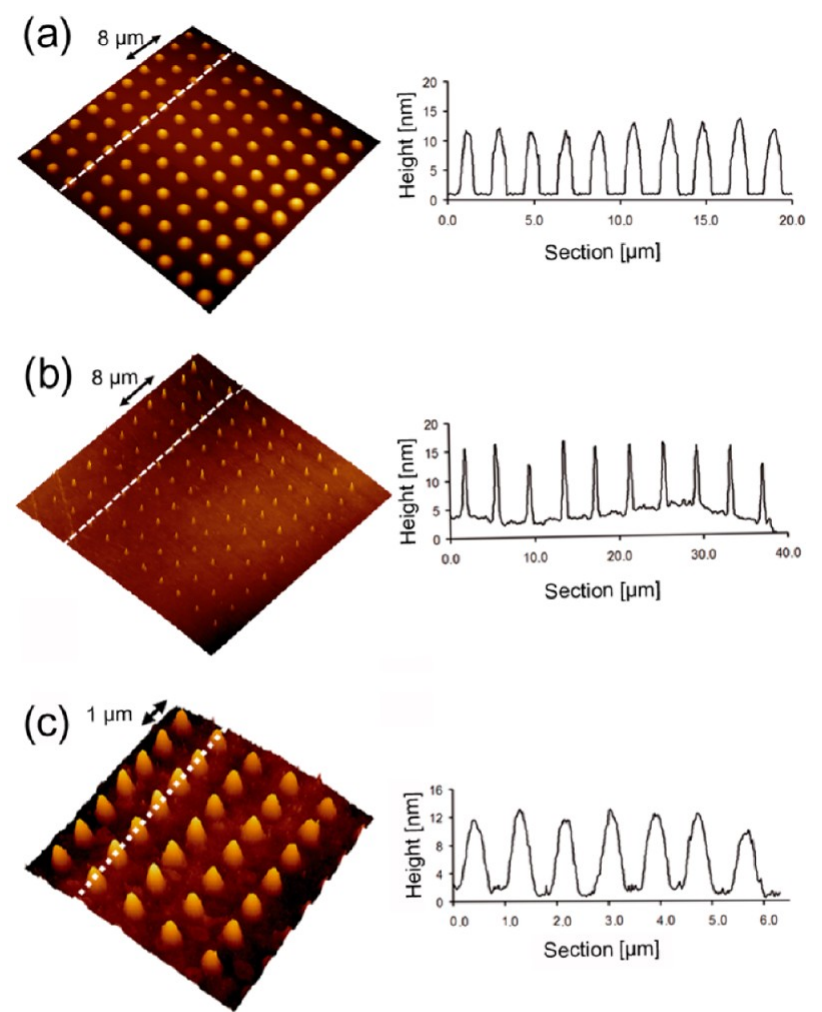

(d)

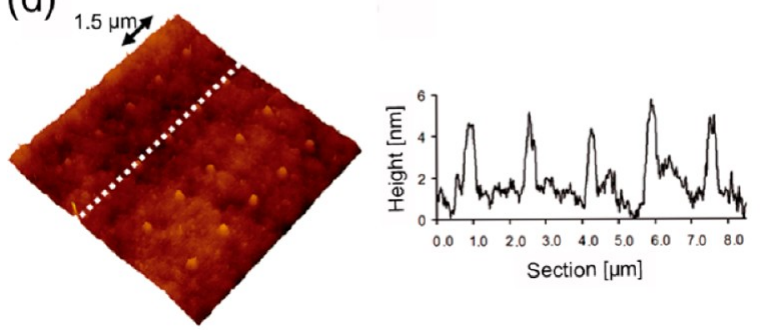

Figure 3. Fabrication of arrays on $\mathrm{Si} / \mathrm{SiO}_{2}$ substrates: (left) 3-D AFM topography image and (right) height profile along the white dashed line in the corresponding image. The specifications for each particular case are (a) ink composition: Co-NPs $\left(2 \times 10^{15} \mathrm{NPs} \mathrm{mL}^{-1}\right)$ in toluene with an excess of oleic acid $(0.08 \% \mathrm{w} / \mathrm{w})$, array of $10 \times 10$ dots separated by $2 \mu \mathrm{m}$, dot diameter $1.01 \pm 0.10 \mu \mathrm{m}$ and height $11 \pm 1$

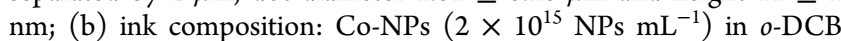
with an excess of oleic acid $(0.08 \% \mathrm{w} / \mathrm{w})$, array of $10 \times 10$ dots separated by $4 \mu \mathrm{m}$, dot diameter $981 \pm 47 \mathrm{~nm}$ and height $12 \pm 1 \mathrm{~nm}$; (c) ink composition: Co-NPs $\left(2 \times 10^{15} \mathrm{NPs} \mathrm{mL}^{-1}\right)$ in $o$-DCB with an excess of oleic acid $(0.08 \% \mathrm{w} / \mathrm{w})$, array of $7 \times 5$ dots separated by 1 $\mu \mathrm{m}$, dot diameter $559 \pm 29 \mathrm{~nm}$ and height $10 \pm 1 \mathrm{~nm}$; (d) ink composition: NP-free solution of $o$-DCB with an excess of oleic acid $(0.08 \% \mathrm{w} / \mathrm{w})$, array of $5 \times 4$ dots separated by $1.5 \mu \mathrm{m}$, dot diameter $473 \pm 51 \mathrm{~nm}$ and height $3 \pm 1 \mathrm{~nm}$.

wettability of our ink. As can be seen in Figure 4, the average dot diameter of the structures generated on $\mathrm{Si} / \mathrm{SiO}_{2}$ was about $\sim 530 \mathrm{~nm}$ whereas those obtained on graphene oscillate around $\sim 675 \mathrm{~nm}$. Even though the wetting capacity of the ink on the graphene surface is slightly higher than on the $\mathrm{Si} / \mathrm{SiO}_{2}$ according to the larger diameter of the resulting dots, the writing capabilities of the ink turn out to be similar for both cases. Since dots were generated in a consecutive manner, possible effects of depletion of the ink were neglected. These results indicate that the graphene flake is strongly influenced by the underneath polar $\mathrm{Si} / \mathrm{SiO}_{2}$ layer. To illustrate this effect, a
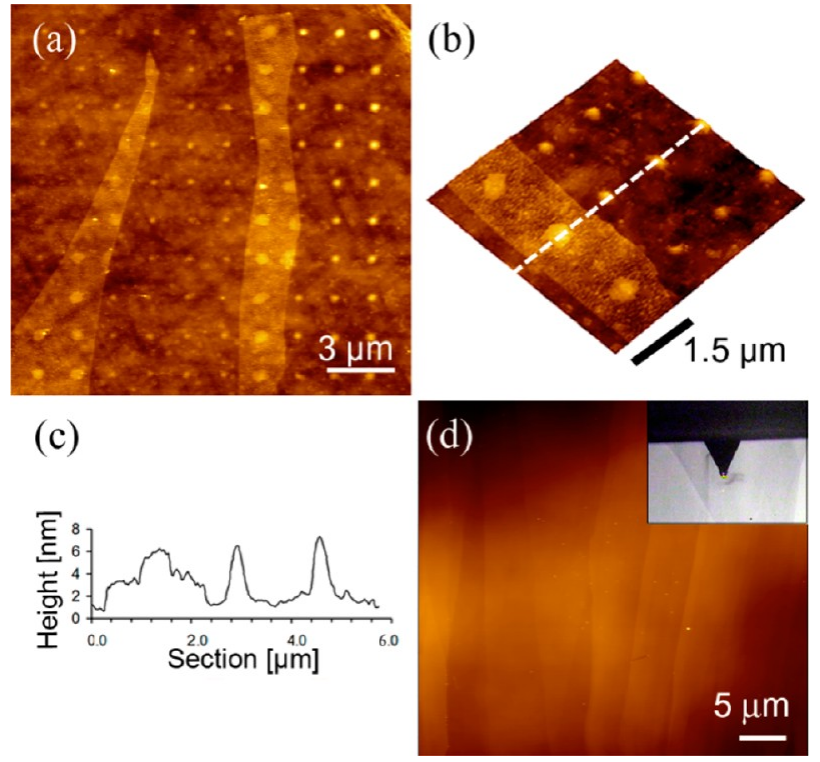

Figure 4. AFM topography images of arrays fabricated on top of three different surfaces, single-layer graphene, $\mathrm{Si} / \mathrm{SiO}_{2}$ and $\mathrm{HOPG}$, by using an NP-free ink solution consisted of $o$-DCB and oleic acid $(0.08 \% \mathrm{w} /$ w). (a) Well-defined arrays were generated on top of graphene singlelayer graphene and $\mathrm{Si} / \mathrm{SiO}_{2}$ surface. (b) 3-D topography of a section of the array shown in image b. (c) Height profile along the white dashed line in the image b. (d) Droplets deposited on HOPG merge to form a thin film $(<3 \mathrm{~nm}$ in height). Inset shows an optical image during the deposition process.

comparative experiment was designed in which the NP-free ink solution was used for the fabrication of arrays on hydrophobic highly ordered pyrolytic graphite (HOPG). The fabrication of patterns on the HOPG surface resulted in a nonconsistent deposition where the droplets tended to spread out almost completely over the whole surface merging into a thin film of less than $3 \mathrm{~nm}$ in height (Figure 4d). The behavior of the droplets can be understood by considering the high affinity of the $o$-DCB to the nonpolar carbon surface.

The presence of such residual thin film is not expected to disturb the effectiveness of the device. For instance, Chen et al. recently reported the fabrication of a highly sensitive and selective field-effect transistor (FET) biosensor using thermally reduced graphene sheets decorated with gold NPs-antibody conjugates. ${ }^{33}$ Under these circumstances, the presence of target proteins induces significant changes on the electrical characteristics of the device detected by the graphene even though the NPs are separated by a capping layer. Another example where the presence of such a layer has been shown to not interfere are fluorescent graphene-based sensors, since it can act as a superquencher with a long-range nanoscale energy transfer property of dyes and QDs. For instance, these types of systems have already been shown to be useful platforms for the sensitive and selective detection of DNA and proteins based on a signalon fluorescent strategy. ${ }^{34}$ In fact, several strategies developed to prepare graphene/metal hybrids take advantage of such "molecular stabilizers" not only because of the better wetting capabilities of the "ink" but especially to ensure a proper stability of the NPs while avoiding any reaction with the graphene. For instance, one of the most popular strategies, the aqueous-phase route, includes the use of capping molecules such as dodecyl sulfate (SDS) as both surfactant and/or reducing agent for the particle formation. ${ }^{35}$ Other method- 
ologies, such as that described by El-Shall's group, involve a facile and scalable chemical reduction method assisted by microwave irradiation in a mixture of oleylamine and oleic acid. $^{36}$

Once the optimal affinity between the ink and the singlelayer graphene supported on $\mathrm{Si} / \mathrm{SiO}_{2}$ was demonstrated, similar conditions were used for the deposition of Co-NPs as consistent arrays onto the graphene layers. A representative example for the formation of such arrays is shown in Figure 5.
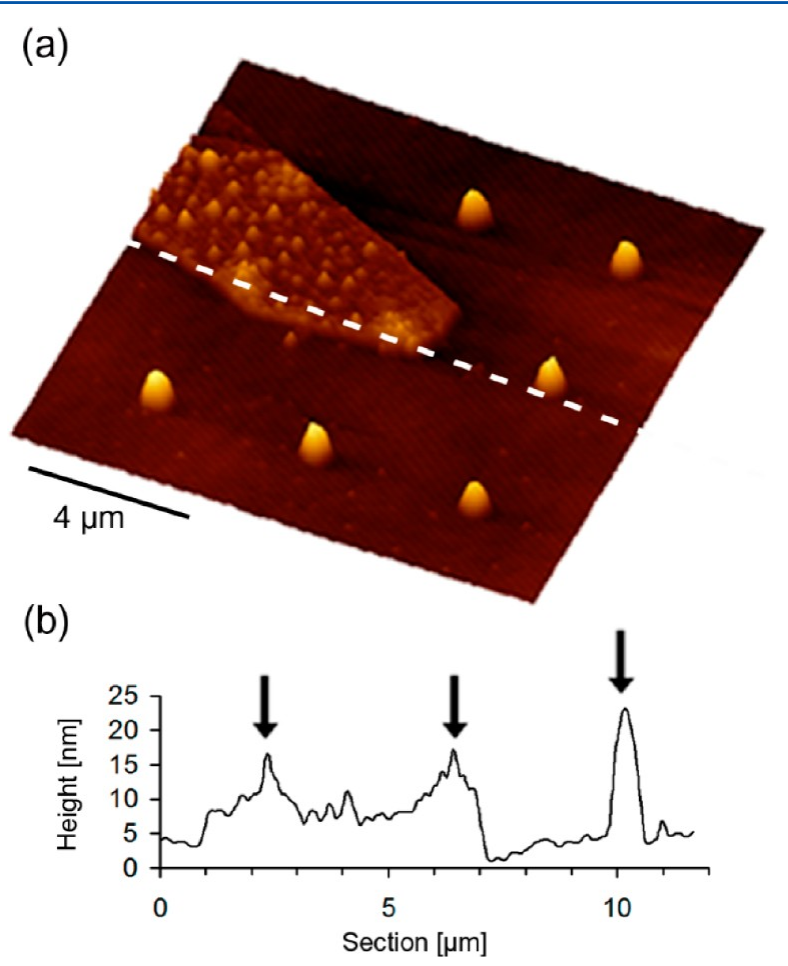

Figure 5. Array containing the Co-NPs generated on graphene and $\mathrm{Si} /$ $\mathrm{SiO}_{2}$ surfaces. Ink composition: Co-NPs $\left(2 \times 10^{15} \mathrm{NPs} \mathrm{mL}^{-1}\right)$ in $o-$ DCB with an excess of oleic acid $(0.08 \% \mathrm{w} / \mathrm{w})$. (a) 3-D AFM topography image and $(\mathrm{b})$ height profile along the white dashed line in the image.

The average dot diameters were $1.35 \mu \mathrm{m}$ and $0.82 \mu \mathrm{m}$ on graphene and $\mathrm{Si} / \mathrm{SiO}_{2}$, respectively, confirming the different wetting capacities of the ink on both surfaces. In addition, the height of the dots considerably increased compared to the NPfree counterpart with an average value of $9 \pm 1 \mathrm{~nm}$ on graphene, in good agreement with the height values expected for a monolayer of NPs.

Another important fact to demonstrate the feasibility of our approach is to verify the integrity of the graphene layer upon deposition. A first confirmation for this is the absence of any evident damaging of graphene after the deposition process by AFM characterization (Figure 4). However, this observation was better proved on successive experiments by combining Raman and electronic transport investigations. In this sense, Raman spectroscopy emerges as a powerful tool to capture graphene electronic structure and thus is uniquely able to reflect changes in graphene after manipulation. ${ }^{37}$ Figure 6a shows Raman spectra taken for a single-layer graphene sample before and after the deposition of NPs. The two main characteristic bands of graphene, the $\mathrm{G}$ band at $\sim 1580 \mathrm{~cm}^{-1}$ and the $2 \mathrm{D}$ band at $\sim 2700 \mathrm{~cm}^{-1}$, which are commonly used in the determination of graphene layer thickness, are well visible (a)
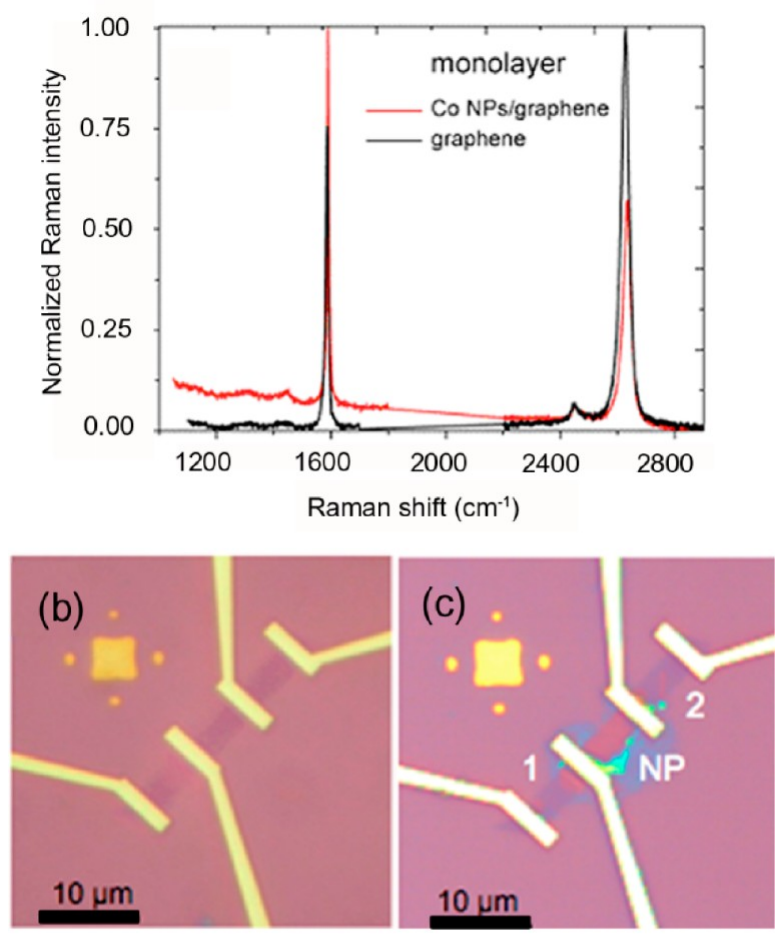

(d)

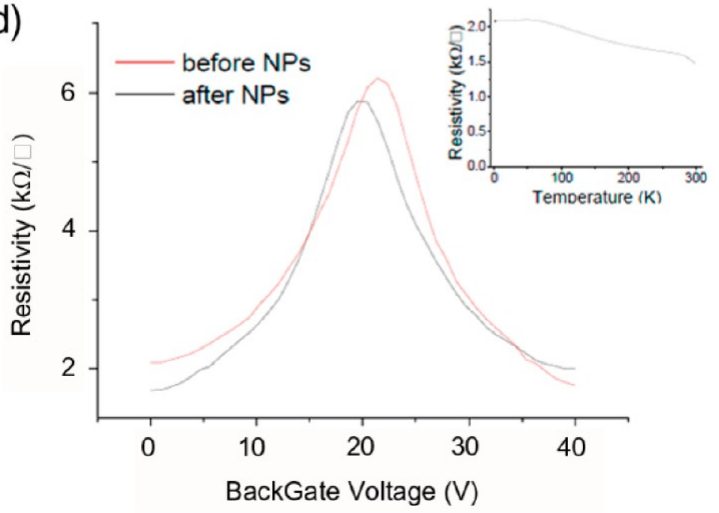

Figure 6. (a) Raman spectra taken before (black) and after (red) deposition of Co-NP arrays on a single-layer graphene. Optical image of an elongated graphene layer decorated with contacts in a longitudinal geometry viewed (b) before and (c) after the deposition of a high-density Co-NP array made of repeated dots separated by 1 $\mu \mathrm{m}$ (blue colored area). (d) Surface resistivity (4 wires measurements) vs backgate voltage of the device shown in image $b$ and $c$. Measurements before (red) and after (black) NPs deposition show the characteristic resistance peak in graphene at the Dirac point. In the inset it is possible to see the temperature dependence of the device resistance at backgate voltage of $0 \mathrm{~V}$.

on the Raman spectrum. The only slight shift of the $G$ band points out to a charge doping effect of graphene due to the presence of Co-NPs. ${ }^{38}$ The absence of the D band at $\sim 1340$ $\mathrm{cm}^{-1}$, typically associated to disorder, indicates that the integrity of graphene was preserved after the deposition step. Finally, the integrity of graphene was also checked by repeating the deposition process on an elongated graphene layer decorated with contacts in a longitudinal geometry (resistance mode) (Figure $6 \mathrm{~b}, \mathrm{c}$ ). Electronic transport measurements were then used to characterize the effective functionalities of graphene devices after the fabrication of arrays (Figure 6d). 
Reproducibility in the resistance vs backgate voltage curve measured at $T=2 \mathrm{~K}$ before and after deposition, showing a mobility of $\mu=2000 \mathrm{~cm}^{2} \mathrm{~V}^{-1} \mathrm{~s}^{-1}$, evidenced that as expected from AFM and Raman investigations graphene properties were not altered after the deposition of the NPs.

3.3. Nanoparticle Assembly. Further insights into the assembly of the Co-NPs within the fabricated dots were obtained by fabricating new arrays on carbon-coated transmission electron microscopy (TEM) grids, which represents a real challenge in nanopatterning nowadays. Such arrays allowed us for the simultaneous morphological characterization of the deposits by complementary AFM and TEM techniques (see Figure 7). TEM characterization allows Co-NPs to be identified
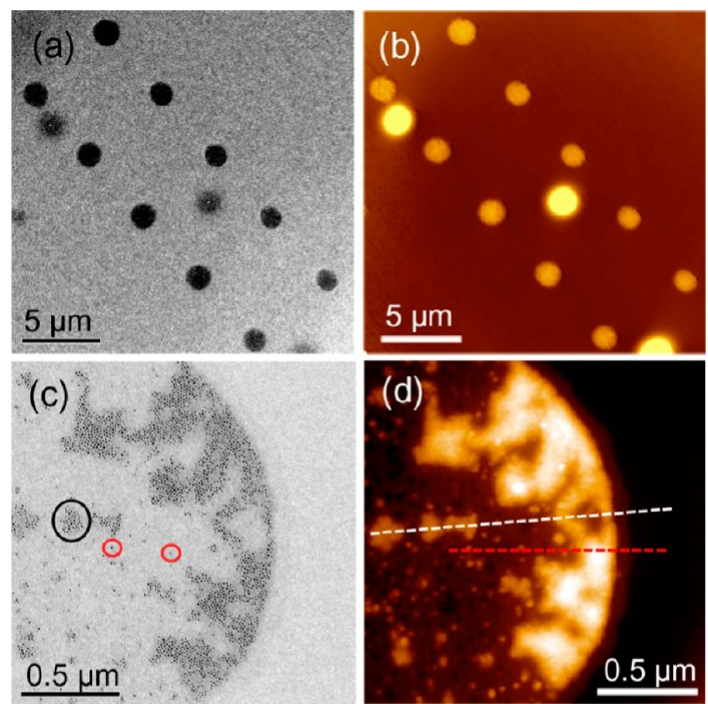

(e) NP island
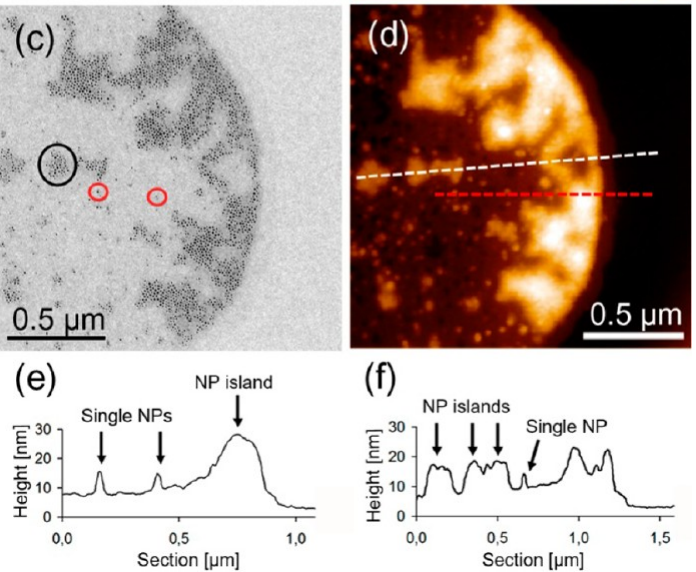

(f)

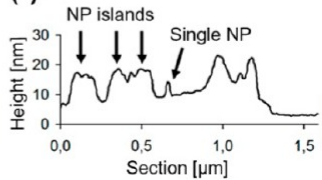

Figure 7. Co-NPs arrays fabricated on carbon-coated TEM grids. (a and b) TEM and AFM topography images of a series of repeated dots. (c and d) TEM and AFM topography images of a section of a dot feature from the array. Height profile in images e and $f$ respectively corresponds to the white and red dashed line in image $d$. The presence of single NPs and NP islands are indicated with black arrows in images $\mathrm{e}$ and $\mathrm{f}$ as well as some examples of these structures are indicated with red and black circles respectively in image $c$.

at the single-particle level due to the high contrast given by the metallic character of the NPs while correlated AFM topography can provide direct information about the topographical profile of the motives. As shown in Figure 7a,c, TEM revealed the presence of NPs as (sub)monolayers inside each dot-like feature that confirmed the effective transference of the ink from the coated tip to the carbon surface. ${ }^{39}$ Within the dots, NPs appeared arranged into hexagonal ordered assemblies (islandlike assemblies), as previously observed for a macroscopically deposited sample (see Figure 1), or isolated particles preferentially at the edge of the dots (Figure 7c) due to the coffee-stain phenomenon that induces droplets to dry in the form of ring-like patterns. ${ }^{40}$

The topographic profile of exactly the same dots than those studied by TEM was obtained by means of AFM characterization. The study of at least 20 selected motifs associated with isolated particles, such as the ones marked with a red circle in Figure $7 \mathrm{c}$, confirmed an average height of $9 \pm 1 \mathrm{~nm}$ (Figure $7 \mathrm{e}, \mathrm{f})$. As expected, this value is in good agreement with the size of a single Co-NP. Surprisingly, TEM images of the island-like assemblies, such as the ones marked with a black circle in Figure $7 c$, revealed a topographic profile for the (sub)monolayers up to $30 \mathrm{~nm}$ in height (Figure 7e,f). This observation suggests specifically for this case an unexpected accumulation of remaining oleic acid molecules during the evaporation process by comparison with those previously observed for $\mathrm{Si} / \mathrm{SiO}_{2}$ and graphene that could be tentatively attributed also to coffee-stain phenomena.

3.4. Localized Positioning. Finally, the feasibility of using an AFM tip for the delivery of NPs was combined with its inherent nanoscale positioning capability to tackle the challenge to dispense them on site-specific graphene structures with (sub)micrometric control. For this, a novel three-step deposition methodology, which is schematically depicted in Figure 8, was initially planned. The first step involves the coating of a tip with the ink solution on an auxiliary $\mathrm{Si} / \mathrm{SiO}_{2}$ substrate by the scanning coating method. Second, the freshly coated tip is positioned in the vicinity of the target area and scanned in noncontact dynamic mode to find a mark that serves as a reference point. Then, the coordinates of the target area with respect the reference point are extracted from an AFM topography image of the graphene previously obtained using an uncoated tip. Finally, the ink loaded tip is positioned on these exact coordinates and brought into contact to the surface to transfer the ink material on top of the target region. Let us note that the two last steps of the process should be performed under feedback control to guarantee the positioning of the tip during the experiment.

This strategy requires the tip to accomplish two main requirements: (I) it should be appropriate for obtaining a high resolution image of the cross area while preventing any damage of graphene and (II) it should be able to appropriately deliver the NPs by matching the size of the generated structure to the dimensions of the motive. Both conditions are met in the AFM probes named noncontact high-resonance frequency $(\mathrm{NCH})$ tips. Due to its high stiffness constant they are designed to work on noncontact dynamic mode favoring the mapping process without producing any damage to the graphene layer. On the contrary, these tips are not specially designed for long writing purposes, as the previously described SP tips, due to their asymmetric pyramidal shape that diminishes the width of the meniscus formed at the tip/surface contact and the lack of a recessed area surrounding the tip base that provides a higher ink material loading (see the Experimental Section for details). However, despite this limitation, $\mathrm{NCH}$ tips could be loaded with enough ink to ensure the delivery of a few (sub)micrometric in-size droplets of the ink in a very reproducible manner by using the scanning coating functionalization method. In addition, it is also important to confirm that good transfer can be done at very low forces to prevent graphene surface damage. To demonstrate this, a series of four dots were generated on $\mathrm{Si} / \mathrm{SiO}_{2}$ with the same $\mathrm{NCH}$ tip under different tip-substrate contact forces (see Figure $9 \mathrm{~b}$ ). As an example, an AFM topography image of a series of four consecutive dots generated at contact force of $\sim 40 \mathrm{nN}$ are shown in Figure 9 .

To evaluate the feasibility of the strategy, the alignment procedure and deposition parameters were initially investigated on a lithographically cross-shaped mark $16 \times 16 \mu \mathrm{m}^{2}$ in size created on $\mathrm{Si} / \mathrm{SiO}_{2}$ substrates. The objective was to specifically 

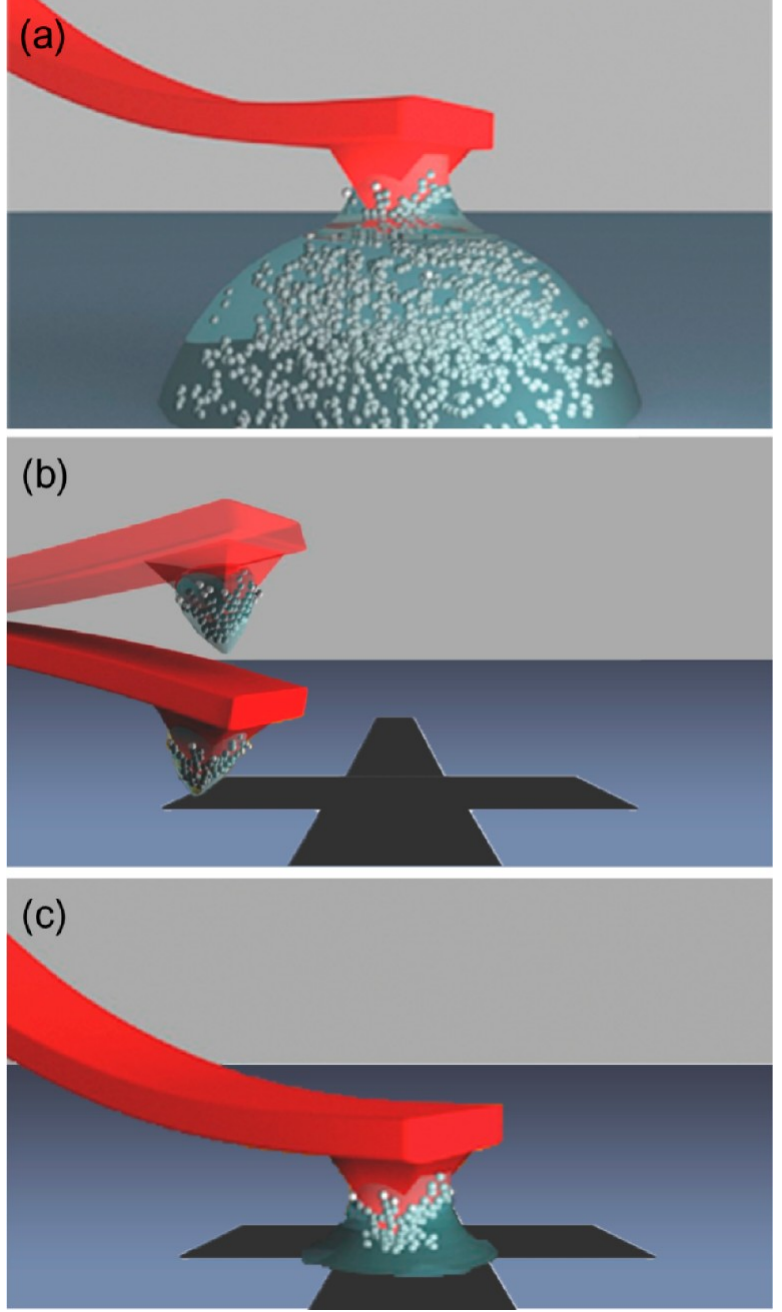

Figure 8. Schematic illustration of a strategy developed for the sitespecific deposition of Co-NPs on a cross-shaped patterned graphene flake with a target area of $1 \times 1 \mu \mathrm{m}^{2}$. (a) An uncoated tip is coated by scanning coating method into a droplet located on an auxiliary $\mathrm{Si} / \mathrm{SiO}_{2}$ surface. (b) The coated tip is positioned in the vicinity of the graphene cross and scanned in noncontact dynamic mode to find a reference point. (c) The graphene cross coordinates with respect the reference point are calculated from an AFM image previously obtained with an uncoated tip. The coated tip is positioned on the target coordinates and the Co-NPs are deposited on the graphene cross.
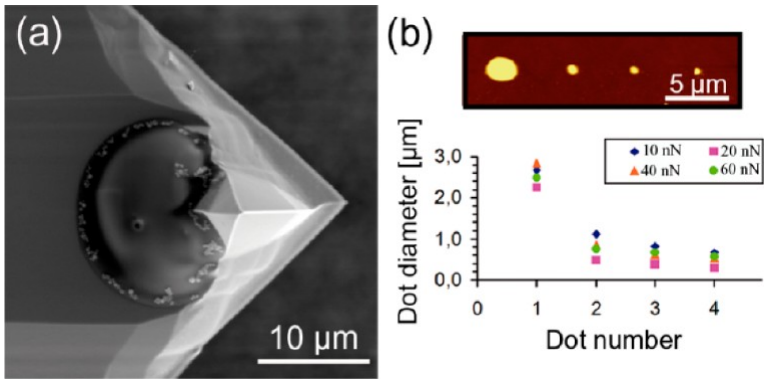

Figure 9. FE-SEM image of a $\mathrm{NCH}$ tip loaded with the ink solution [Co-NPs $\left(2 \times 10^{15} \mathrm{NPs} \mathrm{mL} \mathrm{mL}^{-1}\right)$ in $o$-DCB with an excess of oleic acid $(0.08 \% \mathrm{w} / \mathrm{w})]$. (b) Evolution of the dot diameter according to the ink depletion of a coated $\mathrm{NCH}$ tip. The represented series of four dots on $\mathrm{Si} / \mathrm{SiO}_{2}$ were generated with the same tip but under different tipsubstrate contact forces. Inset shows an AFM topography image of a series of four consecutive dots generated at contact force of $\sim 40 \mathrm{nN}$. deposit the NPs with (sub)micrometric control at the four imaginary points marked with red circles on the AFM image shown in Figure 10a. For this, first the $\mathrm{NCH}$ tip was coated on
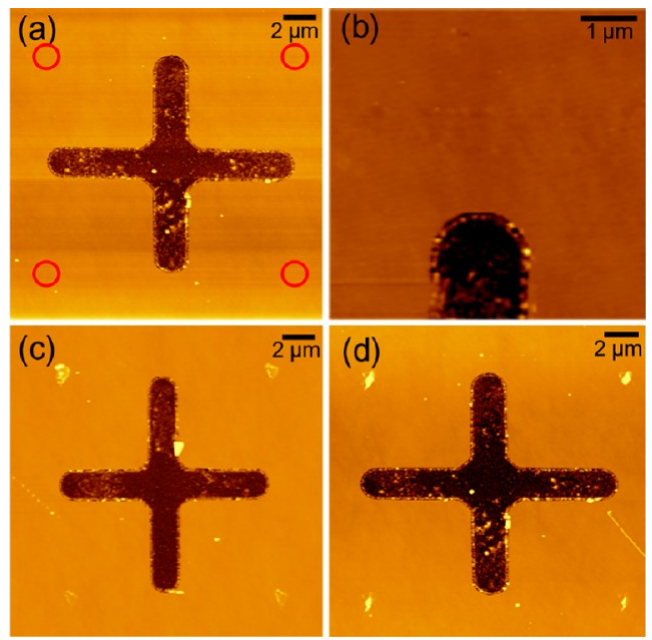

Figure 10. AFM topography images of site-specific deposition experiments performed following the strategy depicted in Figure 8 . (a) Cross-shaped position labels lithographically generated on $\mathrm{Si} / \mathrm{SiO}_{2}$ on which imaginary red circles indicate four regions preselected to localize the deposition of NPs inside. (b) Topography image generated with the coated tip to find the reference point used to calculate the coordinates of the areas on which the deposition should take place (red circles indicated in image a). ( $c$ and d) Two examples of crosses after the deposition of Co-NPs on the preselected positions.

an auxiliary $\mathrm{Si} / \mathrm{SiO}_{2}$ substrate by the scanning coating. Afterward, the freshly coated tip is positioned in the crossshaped mark and scanned in noncontact dynamic mode to obtain the coordinates of reference point (Figure 10b). In this sense, a clean topographic image can be obtained still with a freshly coated tip by keeping the amplitude modulation mode in true attractive regime, with real noncontact working mode. From here, the tip is moved to the four selected deposition areas, whose coordinates with respect the cross-section are previously recorded, and brought into contact to the surface to transfer the ink material at a contact force of $\sim 10 \mathrm{nN}$ during 10 s. Figure 10c,d shows AFM images of two different crosses in which structures matching the four targeted areas surrounding the cross marks were generated in this way. The dots obtained in both examples exhibit an ellipsoidal shape with average axis of $575 \times 1.27 \mu \mathrm{m}^{2}$ and $1.02 \times 1.28 \mu \mathrm{m}^{2}$ as well as heights that oscillate around 47 and $35 \mathrm{~nm}$, respectively. However, even though the fabricated dots were successfully localized on the desired positions of the surface, it is important to stress the poor quality of the depositions, which typically were irregularshaped and nonreproducible from experiment to experiment. Indeed, the intermittent contact of the tip during the mapping of the reference points is likely to modify somehow the ink coating giving to a nonhomogeneous transference of the ink from the tip to the surface. Moreover, the scanned area became contaminated by a small amount of ink material as well as nondesired depositions were also observed over the surface due to the movement of the tip to the different positions (see Figure 10c,d).

To prevent transfer of material from the tip to the substrate during imaging process, it is critical to work in a real attractive regime during dynamic AFM imaging. This is critical to achieve 
while scanning over a graphene substrate due to the high affinity of the ink solvent with the graphene. To overcome this threat, a three-step methodology schematically depicted in Figure 11 was investigated. First, an uncoated tip is used to
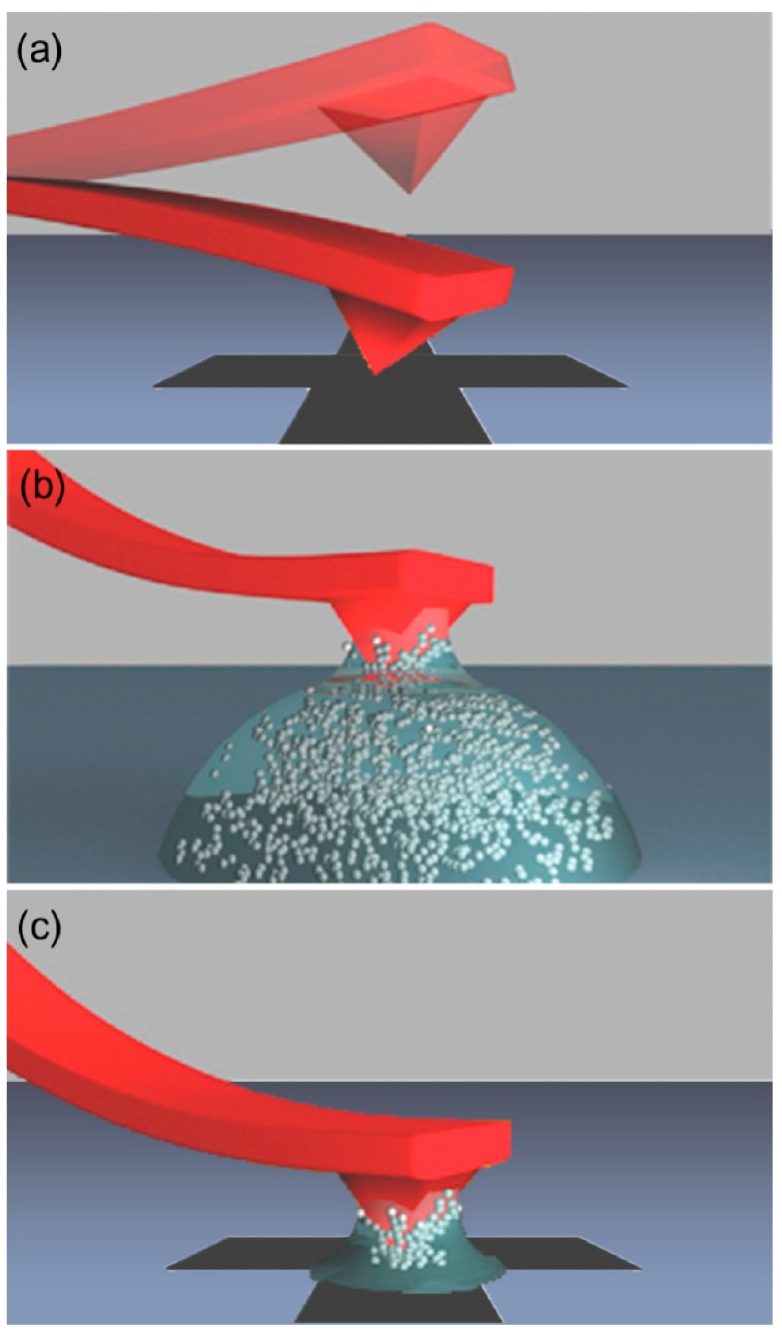

Figure 11. Schematic illustration of the three-step methodology developed for the site-specific deposition of Co-NPs on a cross-shaped patterned graphene flake with a target area of $1 \times 1 \mu \mathrm{m}^{2}$. (a) An uncoated tip is used to obtain a map of the sensor in noncontact dynamic mode and register the coordinates of the target area. (b) The same tip is coated by scanning coating method into a droplet located in the vicinity of the cross. (c) The coated tip is positioned on the registered coordinates and the Co-NPs are deposited on the graphene cross.

obtain an image of the target region, registering the exact coordinates of the cross area of the graphene. In a second step, the tip is moved to a nearby droplet of the ink solution that contains the NPs and then gently coated by scanning in contact mode while dipping the tip into the droplet (scanning coating method). Thereafter, the tip is lifted and moved back exactly to the recorded coordinates corresponding to the target area for the NP deposition by gentle approaching the tip to the substrate and adjusting both tip-substrate contact force and time of contact in order to ensure the transference of the material without damaging the graphene as well as adjust the dot size to the cross.
With this challenge on mind, deposition right at the center of a cross-shaped patterned graphene flake with a target area of 1 $\times 1 \mu \mathrm{m}^{2}$ was faced as a proof-of-concept. One of the most crucial issues in this methodology is to guarantee the positioning of the tip with (sub)micrometric resolution. For this reason, the three steps of the process must be strictly developed under strict feedback control, reason why the $\mathrm{X}-\mathrm{Y}$ navigation of the tip is restricted to the scan range, which in our specific case is $100 \times 100 \mu \mathrm{m}^{2}$ (see the Experimental Section for details). To ensure this restriction, first a $\sim 20 \mu \mathrm{m}$ indiameter droplet of the ink solution, which serves as ink-well for the functionalization of the $\mathrm{NCH}$ tip, is deposited into the $100 \times 100 \mu \mathrm{m}^{2}$ scanning area nearby the graphene cross by approaching a SP tip functionalized with the ink solution by optically monitoring. Then, the uncoated $\mathrm{NCH}$ tip is first used to obtain the exact coordinates of the cross area of the graphene. Afterward, it is moved to the nearby droplet of the NPs-ink solution and then gently coated by scanning in contact mode a $5 \times 5 \mu \mathrm{m}^{2}$ scan area at 1 line $\cdot \mathrm{s}^{-1}$ while dipping the tip into the droplet. Finally, the tip is lifted and moved back exactly to the recorded coordinates corresponding to the target $1 \times 1$ $\mu \mathrm{m}^{2}$ cross-shaped patterned graphene flake to perform the deposition by gentle approaching the tip to the substrate with a force of less than $10 \mathrm{nN}$ during $10 \mathrm{~s}$.

AFM characterization of the $1 \times 1 \mu \mathrm{m}^{2}$ graphene cross area after integration of Co-NPs confirmed the high accuracy achieved in the deposition experiment (Figure 12). The
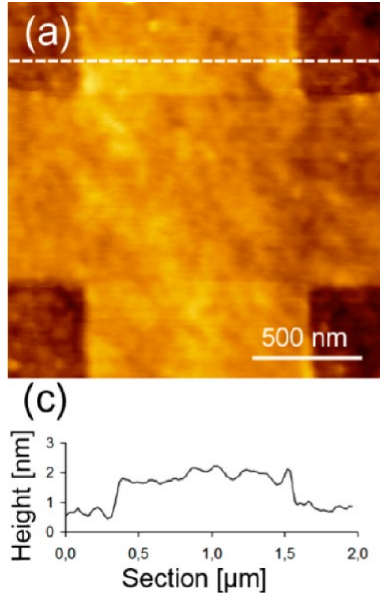

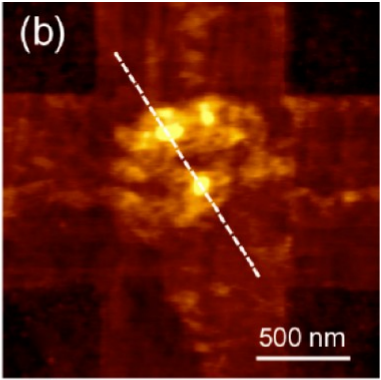

(d)

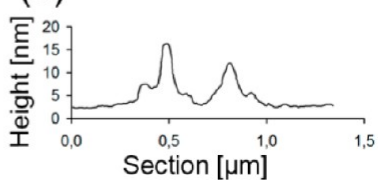

Figure 12. AFM topography image of the cross-shaped patterned graphene flake (cross area of $1 \times 1 \mu \mathrm{m}^{2}$ ) (a) before and (b) after the site-specific deposition of Co-NPs. (c and d) Height profile along the white dashed line in image a and $b$, respectively.

deposited dot exhibits a diameter of $\sim 0.8 \mu \mathrm{m}$ with a basal background that can be up to $6 \mathrm{~nm}$ associated to solid residuals mainly coming from the oleic acid. In addition, different peaks with heights between $10 \mathrm{~nm}$ and $14 \mathrm{~nm}$ and average diameters of $100 \mathrm{~nm}$ to $185 \mathrm{~nm}$ were also observed and associated to the presence of Co-NPs assemblies (Figure 12b,d). A tentative estimation of the number of particles deposited on the cross area from the size (width and height) of the deposit results in approximately 100 Co-NPs. Moreover, the successful deposition over the target area was reinforced by the lack of undesirable depositions during the coated tip movement as confirmed by AFM measurements over larger areas. 


\section{CONCLUSION}

In summary, AFM has been shown to be an excellent lithographic technique to directly positioning NPs on top of graphene by capillary transport without causing any damage. Though the work has been focused on Co-NPs, our experience with DPN aim us to expect this approach to be of universal use for many other nanostructures. ${ }^{39,41-43}$ The deposition takes place without previous functionalization of neither the NPs nor the graphene surface, and with the NPs forming a (sub)monolayer inside the deposited dots. In addition, the experiments have been used to extract valuable basic information on (I) the hydrophilic character of a single-layer graphene supported on $\mathrm{Si} / \mathrm{SiO}_{2}$ by comparison with $\mathrm{HOPG}$ and (II) solvent and surfactant (oleic acid) influence on the tipassisted delivery of an ink as a fixed pattern.

Moreover, accurate control for the positioning of the Co-NPs on preselected sites of a graphene surface has been achieved by evolution of this methodology to a three-step protocol consisting on (I) localization of the target region, (II) functionalization of the tip, and (III) deposition of the desired material, always working under strict feedback conditions. As a proof-of-concept integration of the NPs on a $1 \times 1 \mu \mathrm{m}^{2}$ graphene patterned cross is achieved. These results open new venues for the fabrication of not only hybrid graphene devices but any other device that requires a controlled positioning within nanostructures.

\section{AUTHOR INFORMATION}

\section{Corresponding Author}

*E-mail: druiz@cin2.es.

\section{Notes}

The authors declare no competing financial interest.

\section{ACKNOWLEDGMENTS}

We thank the Spanish government for the project MAT200913977-C03-03. N.D. and E.B. thank the Spanish government for a Ramon y Cajal grant and a FPI grant, respectively. This work has been partially supported by FP7-ICT FET Open “MolSpinQIP" project, Contract No. 211284.

\section{REFERENCES}

(1) Novoselov, K. S.; Geim, A. K.; Morozov, S. V.; Jiang, D.; Zhang, Y.; Dubonos, S. V.; Grigorieva, I. V.; Firsov, A. A. Electric Field Effect in Atomically Thin Carbon Films. Science 2004, 306, 666-669.

(2) Novoselov, K. S.; Geim, A. K.; Morozov, S. V.; Jiang, D.; Katsnelson, M. I.; Grigorieva, I. V.; Dubonos, S. V.; Firsov, A. A. Twodimensional gas of massless Dirac fermions in graphene. Nature 2005, 438, 197-200.

(3) Geim, A. K.; Novoselov, K. S. The rise of graphene. Nat. Mater. 2007, 6, 183-191.

(4) Balandin, A. A.; Ghosh, S.; Bao, W.; Calizo, I.; Teweldebrhan, D.; Miao, F.; Lau, C. N. Superior Thermal Conductivity of Single-Layer Graphene. Nano Lett. 2008, 8, 902-907.

(5) Geim, A. K. Graphene: Status and Prospects. Science 2009, 324, $1530-1534$.

(6) Lee, C.; Wei, X.; Kysar, J. W.; Hone, J. Measurement of the Elastic Properties and Intrinsic Strength of Monolayer Graphene. Science 2008, 321, 385-388.

(7) Guo, S.; Dong, S. Graphene nanosheet: synthesis, molecular engineering, thin film, hybrids, and energy and analytical applications. Chem. Soc. Rev. 2011, 40, 2644-2672.

(8) Bonaccorso, F.; Sun, Z.; Hasan, T.; Ferrari, A. C. Graphene photonics and optoelectronics. Nat. Photon. 2010, 4, 611-622.
(9) Castro Neto, A. H.; Guinea, F.; Peres, N. M. R.; Novoselov, K. S.; Geim, A. K. The electronic properties of graphene. Rev. Mod. Phys. 2009, 81, 109-162.

(10) Tombros, N.; Jozsa, C.; Popinciuc, M.; Jonkman, H. T.; van Wees, B. J. Electronic spin transport and spin precession in single graphene layers at room temperature. Nature 2007, 448, 571-574.

(11) Lopes, M.; Candini, A.; Urdampilleta, M.; Reserbat-Plantey, A.; Bellini, V.; Klyatskaya, S.; Marty, L. t.; Ruben, M.; Affronte, M.; Wernsdorfer, W.; Bendiab, N. Surface-Enhanced Raman Signal for Terbium Single-Molecule Magnets Grafted on Graphene. ACS Nano 2010, 4, 7531-7537.

(12) Candini, A.; Klyatskaya, S.; Ruben, M.; Wernsdorfer, W.; Affronte, M. Graphene Spintronic Devices with Molecular Nanomagnets. Nano Lett. 2011, 11, 2634-2639.

(13) Huang, X.; Yin, Z.; Wu, S.; Qi, X.; He, Q.; Zhang, Q.; Yan, Q.; Boey, F.; Zhang, H. Graphene-Based Materials: Synthesis, Characterization, Properties, and Applications. Small 2011, 7, 1876-1902.

(14) Scheuermann, G. M.; Rumi, L.; Steurer, P.; Bannwarth, W.; Mülhaupt, R. Palladium Nanoparticles on Graphite Oxide and Its Functionalized Graphene Derivatives as Highly Active Catalysts for the Suzuki-Miyaura Coupling Reaction. J. Am. Chem. Soc. 2009, 131, $8262-8270$.

(15) Cao, A.; Liu, Z.; Chu, S.; Wu, M.; Ye, Z.; Cai, Z.; Chang, Y.; Wang, S.; Gong, Q.; Liu, Y. A Facile One-step Method to Produce Graphene-CdS Quantum Dot Nanocomposites as Promising Optoelectronic Materials. Adv. Mater. 2010, 22, 103-106.

(16) Lin, Y.; Zhang, K.; Chen, W.; Liu, Y.; Geng, Z.; Zeng, J.; Pan, N.; Yan, L.; Wang, X.; Hou, J. G. Dramatically Enhanced Photoresponse of Reduced Graphene Oxide with Linker-Free Anchored CdSe Nanoparticles. ACS Nano 2010, 4, 3033-3038.

(17) Geng, X.; Niu, L.; Xing, Z.; Song, R.; Liu, G.; Sun, M.; Cheng, G.; Zhong, H.; Liu, Z.; Zhang, Z.; Sun, L.; Xu, H.; Lu, L.; Liu, L. Aqueous-Processable Noncovalent Chemically Converted GrapheneQuantum Dot Composites for Flexible and Transparent Optoelectronic Films. Adv. Mater. 2010, 22, 638-642.

(18) Liu, J.; Fu, S.; Yuan, B.; Li, Y.; Deng, Z. Toward a Universal "Adhesive Nanosheet" for the Assembly of Multiple Nanoparticles Based on a Protein-Induced Reduction/Decoration of Graphene Oxide. J. Am. Chem. Soc. 2010, 132, 7279-7281.

(19) Lee, J.; Shim, S.; Kim, B.; Shin, H. S. Surface-Enhanced Raman Scattering of Single- and Few-Layer Graphene by the Deposition of Gold Nanoparticles. Chem.-Eur. J. 2011, 17, 2381-2387.

(20) Schedin, F.; Lidorikis, E.; Lombardo, A.; Kravets, V. G.; Geim, A. K.; Grigorenko, A. N.; Novoselov, K. S.; Ferrari, A. C. SurfaceEnhanced Raman Spectroscopy of Graphene. ACS Nano 2010, 4, 5617-5626.

(21) Huh, S.; Park, J.; Kim, K. S.; Hong, B. H.; Kim, S. B. Selective nType Doping of Graphene by Photo-patterned Gold Nanoparticles. ACS Nano 2011, 5, 3639-3644.

(22) Domingo, N.; Bellido, E.; Ruiz-Molina, D. Advances on structuring, integration and magnetic characterization of molecular nanomagnets on surfaces and devices. Chem. Soc. Rev. 2012, 41, 258302.

(23) Piner, R. D.; Zhu, J.; Xu, F.; Hong, S.; Mirkin, C. A. "Dip-Pen" Nanolithography. Science 1999, 283, 661-663.

(24) Salaita, K.; Wang, Y.; Mirkin, C. A. Applications of dip-pen nanolithography. Nat. Nano 2007, 2, 145-155.

(25) Basnar, B.; Willner, I. Dip-Pen-Nanolithographic Patterning of Metallic, Semiconductor, and Metal Oxide Nanostructures on Surfaces. Small 2009, 5, 28-44.

(26) Puntes, V. F.; Krishnan, K. M.; Alivisatos, A. P. Colloidal Nanocrystal Shape and Size Control: The Case of Cobalt. Science 2001, 291, 2115-2117.

(27) Warner, J. H.; Rummeli, M. H.; Bachmatiuk, Z. A.; Wilson, M.; Buchner, B. Examining Co-Based Nanocrystals on Graphene Using Low-Voltage Aberration-Corrected Transmission Electron Microscopy. ACS Nano 2010, 4, 470-476. 
(28) Wang, W. M.; Stander, N.; Stoltenberg, R. M.; GoldhaberGordon, D.; Bao, Z. Dip-Pen Nanolithography of Electrical Contacts to Single Graphene Flakes. ACS Nano 2010, 4, 6409-6416.

(29) Li, B.; Goh, C. F.; Zhou, X.; Lu, G.; Tantang, H.; Chen, Y.; Xue, C.; Boey, F. Y. C.; Zhang, H. Patterning Colloidal Metal Nanoparticles for Controlled Growth of Carbon Nanotubes. Adv. Mater. 2008, 20, 4873-4878.

(30) Ben Ali, M.; Ondarçuhu, T.; Brust, M.; Joachim, C. Atomic Force Microscope Tip Nanoprinting of Gold Nanoclusters. Langmuir 2002, 18, 872-876.

(31) Hamilton, C. E.; Lomeda, J. R.; Sun, Z.; Tour, J. M.; Barron, A. R. High-Yield Organic Dispersions of Unfunctionalized Graphene. Nano Lett. 2009, 9, 3460-3462.

(32) Courty, A.; Fermon, C.; Pileni, M. P. Supra Crystals" Made of Nanocrystals. Adv. Mater. 2001, 13, 254-258.

(33) Mao, S.; Lu, G.; Yu, K.; Bo, Z.; Chen, J. Specific Protein Detection Using Thermally Reduced Graphene Oxide Sheet Decorated with Gold Nanoparticle-Antibody Conjugates. Adv. Mater. 2010, 22, 3521-3526.

(34) Lu, C.-H.; Yang, H.-H.; Zhu, C. -L.; Chen, X.; Chen, G.-N. A Graphene Platform for Sensing Biomolecules. Angew. Chem., Int. Ed. Engl. 2009, 48, 4785-4787.

(35) Li, Y.; Fan, X.; Qi, J.; Ji, J.; Wang, S.; Zhang, G.; Zhang, F. Palladium Nanoparticle-Graphene Hybrids as Active Catalysts for the Suzuki Reaction. Nano Res. 2010, 3, 429-437.

(36) Hassan, H. M. A.; Abdelsayed, V.; Khder, A. S.; AbouZeid, K. M.; Terner, J.; Samy El-Shall, M.; Al-Resayes, S. I.; El-Azhary, A. A. J. Mater. Chem. 2009, 19, 3832-3837.

(37) Ferrari, A. C.; Meyer, J. C.; Scardaci, V.; Casiraghi, C.; Lazzeri, M.; Mauri, F.; Piscanec, S.; Jiang, D.; Novoselov, K. S.; Roth, S.; Geim, A. K. Raman spectrum of graphene and graphene layers. Phys. Rev. Lett. 2006, 97, 187401.

(38) Yan, J.; Zhang, Y.; Kim, P.; Pinczuk, A. Electric Field Effect Tuning of Electron-Phonon Coupling in Graphene. Phys. Rev. Lett. 2007, 98, 166802 .

(39) Bellido, E.; de Miguel, R.; Ruiz-Molina, D.; Lostao, A.; Maspoch, D. Controlling the Number of Proteins with Dip-Pen Nanolithography. Adv. Mater. 2010, 22, 352-355.

(40) Deegan, R. D.; Bakajin, O.; Dupont, T. F.; Huber, G.; Nagel, S. R; Witten, T. A. Capillary flow as the cause of ring stains from dried liquid drops. Nature 1997, 389, 827-829.

(41) Martínez-Otero, A.; González-Monje, P.; Maspoch, D.; Hernando, J.; Ruiz-Molina, D. Multiplexed arrays of chemosensors by parallel dip-pen nanolithography. Chem. Commun. 2011, 47, 68646866.

(42) Bellido, E.; Cardona-Serra, S.; Coronado, E.; Ruiz-Molina, D. Assisted-assembly of coordination materials into advanced nanoarchitectures by Dip Pen nanolithography. Chem. Commun. 2011, 47, $5175-5177$.

(43) Martínez-Otero, A.; Hernando, J.; Ruiz-Molina, D.; Maspoch, D. $\mathrm{pH}$-responsive fluorescent nanoarrays fabricated by direct-write parallel dip-pen nanolithography. Small 2008, 4, 2131-2135. 